\title{
USO DA TÉCNICA DE RESISTOGRAFIA E DE VARIÁVEIS DENDROMÉTRICAS NA MODELAGEM DA DENSIDADE BÁSICA DE POVOAMENTOS CLONAIS DE Eucalyptus
}

\author{
USE OF RESISTOGRAPH TECHNIQUE AND OF DENDROMETRIC VARIABLES IN THE \\ MODELING OF THE BASIC DENSITY OF CLONAL POPULATIONS Eucalyptus
}

\author{
Donizete da Costa Dias ${ }^{1}$ Jorge Luiz Colodette ${ }^{2}$ Claudio Roberto Thiersch ${ }^{3}$ Hélio Garcia Leite ${ }^{4}$ \\ José Livio Gomide ${ }^{2}$
}

\begin{abstract}
RESUMO
A densidade básica é uma propriedade-chave de qualidade da madeira para produção de celulose, mas sua determinação demanda muito tempo e altos custos. $\mathrm{O}$ desenvolvimento de ferramentas de amostragem não destrutiva, eficiente e de baixo custo torna-se importante, e o Resistógrafo pode ser uma alternativa. Este estudo teve o objetivo de utilizar dados de amplitude de resistência à perfuração coletados pelo Resistógrafo, associados a variáveis dendrométricas, para desenvolver modelos para estimativa de densidade básica em povoamentos clonais de Eucalyptus. Utilizaram-se quatro clones híbridos Eucalyptus grandis x Eucalyptus urophylla distribuídos em plantios comerciais no Vale do Paraíba com idade variando de 2 a 7 anos. Os materiais foram amostrados pelo Resistógrafo a $1,30 \mathrm{~m}$ do solo (DAP) e pela retirada de um disco na mesma altura para determinação da densidade básica em laboratório. Foram selecionadas equações lineares simples e múltiplas, iniciando pelo uso de dados do Resistógrafo (amplitude média) como variável preditora, e, posteriormente incluindo variáveis dendrométricas como DAP, idade, altura total, altura média das árvores dominantes e diâmetro médio quadrático. A inclusão destas variáveis aumentou a precisão das estimativas. A melhor equação selecionada para o conjunto dos 4 clones, ajustada, utilizando a amplitude média (Resistógrafo), associada à altura média das árvores dominantes, à idade das árvores e ao diâmetro médio quadrático, apresentou $\mathrm{R}_{\text {aj }}^{2}$ igual a $68,80 \%$, com erro padrão residual de $0,0201 \mathrm{~g} / \mathrm{cm}^{3}$ ou $4,31 \%$. Para cada clone, isoladamente, as equações mais precisas também foram aquelas em que se associou o Resistógrafo às variáveis do indivíduo e da população. Equações ajustadas em função apenas de variáveis dendrométricas apresentaram medidas de precisão superiores às ajustadas em função apenas do Resistógrafo, no caso do conjunto dos 4 clones, e superiores ao Resistógrafo associado a variáveis do indivíduo, no caso de cada clone isoladamente. O Resistógrafo mostrou-se como uma ferramenta eficiente para predição da densidade básica. Entretanto, estimativas tão ou mais precisas podem ser obtidas sem uso do equipamento quando se têm variáveis individuais e da população.
\end{abstract}

Palavras-chave: densidade básica; Eucalyptus; resistógrafo; amostragem não destrutiva.

\section{ABSTRACT}

The basic density is a key property of wood quality for pulp production, but its determination is very

1 Biólogo, MSc., Coordenador de Melhoramento Genético Florestal, Centro de Tecnologia da Fibria Celulose SA, Rod. Gal. Euryale de Jesus Zerbine, km 84 - SP 66, São Silvestre, CEP 12340-010, Jacareí (SP), Brasil. donizete.dias@fibria.com.br

2 Engenheiro Florestal, PhD., Professor Titular do Departamento de Engenharia Florestal, Universidade Federal de Viçosa, Laboratório de Celulose e Papel, Campus UFV, CEP 36570-000, Viçosa (MG), Brasil. colodett@ufv.br/ jlgomide@ufv.br

3 Engenheiro Florestal, Dr., Professor do Departamento de Engenharia Florestal, Universidade Federal de São Carlos, Campus Sorocaba, Rod. João Leme dos Santos, Km 110, CEP 18052-780, Sorocaba (SP), Brasil. crthiersch@ufscar.br

4 Engenheiro Florestal, Dr., Professor do Departamento de Engenharia Florestal, Universidade Federal de Viçosa, Centro de Ciências Agrárias, Campus UFV, CEP 36570-000, Viçosa (MG), Brasil. hgleite@ufv.br

Recebido para publicação em 22/01/2011 e aceito em 10/11/2015 
time consuming and costly. The development of non-destructive sampling tools, efficient and low cost is important so the Resistograph can be an alternative. This study aimed to use drilling resistance amplitude data collected by the Resistograph, associated to dendrometrical variables, to develop models to estimate basic density in Eucalyptus clonal stands. It was used four hybrid clones of Eucalyptus grandis $\mathrm{x}$ Eucalyptus urophylla distributed in commercial plantations in Paraíba valley, with aging from 2 to 7 years. These materials were sampled by the Resistograph at $1.30 \mathrm{~m}$ above ground (DBH) and the removal of a disk at the same height to determine the density in the laboratory. Single and multiple linear equations were selected starting with data usage by the Resistograph (amplitude) as a predictor variable, and then dendrometric variables such as $\mathrm{DBH}$, age, height, average height of dominant trees and quadratic mean diameter. The inclusion of these variables increased the accuracy of estimates. The best equation selected for all four clones, adjusted using the average amplitude (the Resistograph), associated with the average height of dominant trees, the tree age and quadratic mean diameter, presented $\mathrm{R}^{2}$ aj equal to $68.80 \%$, with error residual standard $0.0201 \mathrm{~g} / \mathrm{cm}^{3}$ or $4.31 \%$. For each clone alone, the more accurate equations also were those involving the Resistograph variables of the individual and the population. Equations adjusted for only dendrometric variables showed precision measurements higher than the ones adjusted for only the Resistograph, in the case of all four clones, and superior to the Resistograph associated with the individual variables in the case of each clone alone. The Resistograph proved to be an efficient tool for the prediction of density. However, equally or more accurate estimates can be obtained without use of the equipment when you have individual variables and population.

Keywords: basic density; Eucalyptus; resistograph; non-destructive sampling.

\section{INTRODUÇÃO}

A densidade básica é uma das características mais importantes da madeira devido a sua relação com o rendimento das operações florestais e dos processos industriais. De forma geral, ela é definida como sendo a quantidade da substância madeira presente em um dado volume, obtido pela razão entre a massa seca e o seu volume verde.

Santos (2002) define a densidade básica como resultante da interação entre propriedades químicas e anatômicas da madeira, ressaltando sua influência no processo de polpação e nos parâmetros de qualidade da polpa. Queiroz et al. (2004) comentam que a avaliação adequada da densidade básica fornece indicações bastante precisas sobre a impregnação dos cavacos e sobre o rendimento do processo de polpação e que geralmente está associada às características físico-mecânicas da polpa e a sua qualidade. Trabalhando com 10 clones de Eucalyptus selecionados por empresas brasileiras, Lopez, Gomide e Phillips (2009) concluíram que a densidade básica, juntamente com o incremento volumétrico médio anual e o teor de lignina, influenciam significativamente no valor presente líquido (VPL) do complexo integrado pela floresta e a fábrica de celulose. Esses estudos corroboram as afirmações de Gomes (2007), de que a densidade básica é uma propriedade-chave para a produção de celulose, e de Foelkel, Mora e Menochelli (1990) de que é um bom índice para avaliar a qualidade da madeira.

Segundo Hansen (2000) e Lopes e Garcia (2002), a densidade básica da madeira possui um caráter muito complexo e suas variações estão relacionadas com as características anatômicas da madeira, como comprimento e largura da célula, espessura da parede celular e diâmetro do lume, além das proporções de lenho inicial e lenho tardio e os teores de celulose, lignina e extrativos.

Apesar da importância do conhecimento da densidade básica da madeira a ser utilizada no processo industrial, as técnicas tradicionais de sua determinação apresentam alguns inconvenientes operacionais que podem limitar o atendimento satisfatório à indústria. Os principais são o baixo rendimento da operação (amostragem, transporte, preparo de amostras e determinação da densidade básica em laboratório), e o alto custo da atividade. Esses fatores levam a uma amostragem reduzida que por vezes não abrange as variações ambientais e genotípicas da população avaliada.

Dada a importância e a dificuldade de obtenção da densidade básica, muitos estudos têm sido feitos na tentativa de encontrar metodologias de amostragem mais simples e menos onerosas, passando quase sempre pelas amostragens não destrutivas. Os trabalhos de Rinn, Schweingruber e Schar (1996), Hansen (2000), Trugilho et al. (2006), Lima et al. (2007) e Gomes (2007), são alguns exemplos. Sobre este aspecto, 
Thiersch et al. (2006) ressaltam que a densidade básica pode ser relacionada a outras características de fácil medição, podendo, portanto, ser modelada. Os mesmos autores citam que o controle da estrutura do povoamento (diâmetro e altura), da idade, da capacidade produtiva dos locais, do manejo e dos materiais genéticos, somados a alguma variável mecânica permite construir modelos precisos para predição da densidade básica média da árvore.

Neste sentido, uma das ferramentas para obtenção de uma variável mecânica de fácil medição e não destrutiva é o Resistógrafo. Segundo Rinn, Schweingruber e Schar (1996), o Resistógrafo baseia-se numa broca de aço de $3 \mathrm{~mm}$ de diâmetro, que, ao penetrar na madeira, registra as variações de intensidade de torque correspondentes às variações estruturais da parede celular, lenho inicial e tardio e efeitos das condições de crescimento da árvore. Os registros são feitos por sensores mecânicos e eletrônicos do equipamento como um valor de resistência à perfuração, denominado amplitude.

O objetivo deste estudo foi utilizar dados de amplitude de resistência à perfuração coletados pelo Resistógrafo, associados a variáveis dendrométricas, para testar e ou desenvolver modelos para estimativa de densidade básica em povoamentos clonais de Eucalyptus.

\section{MATERIAL E MÉTODOS}

\section{Caracterização das populações de estudo}

Para este estudo, foram amostradas árvores em quatro cultivares clonais identificados como C2, C4, P95 e V3, todos híbridos Eucalyptus grandis x Eucalyptus urophylla, implantados em larga escala em áreas distribuídas por todo o Vale do Paraíba, (Leste do Estado de São Paulo e Sul do Estado do Rio de Janeiro), entre as latitudes $22^{\circ} 30^{\prime}$ a $23^{\circ} 30^{\prime} \mathrm{S}$ e longitudes $44^{\circ} 22^{\prime}$ a $46^{\circ} 12^{\prime} \mathrm{W}$. As amostras foram selecionadas buscando a distribuição mais igualitária possível das variações de idade, sítio, altitude, diâmetro a 1,30 m do solo (DAP), altura total, altura média, altura dominante e volume.

As amostras foram coletadas no entorno de parcelas do inventário florestal contínuo, abrangendo idades entre 2 e 7 anos. Em cada parcela foram determinadas 4 classes diamétricas, sendo amostrada uma árvore para cada classe/parcela, totalizando 372 árvores.

As árvores escolhidas foram amostradas pelo Resistógrafo, modelo IML-F400-S, sempre na altura de $1,30 \mathrm{~m}$ do solo (DAP), com o equipamento ajustado para madeiras duras. Nesta mesma posição foi retirado um disco com espessura aproximada de $3 \mathrm{~cm}$ para determinação da densidade básica em laboratório. Em seguida, cada árvore abatida teve seu volume individual determinado por cubagem rigorosa pelo método de Smalian, nas alturas 0,$10 ; 0,70 ; 1,30 ; 2,00 ; 4,00 \mathrm{~m}$ e, assim sucessivamente de 2 em 2 metros até atingir a altura do diâmetro mínimo comercial de $3,00 \mathrm{~cm}$ com casca.

A densidade básica dos discos foi determinada em laboratório pelo método da balança hidrostática, conforme descrito e utilizado por Alzate, Tomazello Filho e Piedade (2005).

\section{Tratamento dos dados de amplitude do Resistógrafo ${ }^{\circledR}$}

O Resistógrafo registra 100 pares de profundidade e amplitude a cada centímetro de avanço da broca no sentido radial do tronco, ou seja, mede a resistência à penetração a cada $0,1 \mathrm{~mm}$. Essa quantidade de pontos de amostragem permite representar com bastante detalhe o perfil de resistência da árvore. Porém, variações inerentes à própria madeira, como presença de nós e bolsas de resina, ou inerentes ao processo de amostragem, tais como passagem da broca pela casca da árvore ou leitura feita com a broca fora da madeira, podem gerar dados não representativos da estrutura do tronco.

Devido a isso, os dados de cada amostra passaram por alguns tratamentos visando à eliminação de valores nulos (zeros) e remoção de dados estranhos (outliers).

\section{Eliminação de leituras fora do lenho}

Foram eliminados os valores de amplitude iguais a "zero", que indicavam movimentação da broca antes de iniciar penetração na árvore e, também, as amplitudes iguais a $0,5 \%$ referentes à passagem da broca 
pela casca da árvore. Após remoção, foi calculada a média geral da amplitude por amostra, denominada AMP_100.

\section{Eliminação de dados extremos}

Para o cálculo de outras médias das resistências à penetração no lenho, foram removidos os valores extremos que podem ocorrer devido a situações diversas, como por exemplo, presença de nós, rachaduras, podridão, bolsas de resina, etc.

Na primeira forma de remoção, para obter, por exemplo, a média denominada AMP_90, foram consideradas todas as amplitudes entre os percentis $5 \% \mathrm{e} 95 \%$. O procedimento foi repetido para a construção das médias das amplitudes entre os percentis 0,5\% a 99,5\% (AMP_99), 2,5\% a 97,5\% (AMP_95) e 5,0\% a 95,0\% (AMP_90).

Como segunda forma de remoção de dados extremos foi aplicado o critério do gráfico de caixa (Boxplot) visando identificar e eliminar dados estranhos de amplitude. Os valores identificados por este critério, denominados outliers, foram excluídos e foi gerada uma nova média de amplitude (A_OUT) para cada árvore.

\section{Modelagem da densidade}

Para a estimativa da densidade básica média da árvore foram desenvolvidos modelos lineares simples ou múltiplos, em que somente a média de amplitude de resistência à perfuração dada pelo Resistógrafo foi utilizada como variável independente, embora com diferentes formas de apresentação dadas pelos tratamentos dos dados.

Em um segundo momento, com o intuito de melhorar a estimativa da densidade básica média da árvore, foram desenvolvidos modelos lineares múltiplos, incluindo variáveis dendrométricas como a altura média das árvores dominantes (HD), o diâmetro a 1,30 m de altura (DAP), a altura total (HT), o diâmetro médio quadrático (DG) e a idade (ID), bem como variações e combinações destas variáveis.

Para a construção dos modelos lineares, foi utilizado o procedimento stepwise. Para cada modelo construído foi analisado o coeficiente de determinação corrigido, em porcentagem $\left(\mathrm{R}_{\mathrm{aj}}^{2}\right)$, o erro padrão residual (Syx) na escala original da variável dependente e em porcentagem e o gráfico dos resíduos.

\section{RESULTADOS E DISCUSSÃO}

\section{Caracterização da base de dados}

As informações básicas da população clonal amostrada são apresentadas na Tabela 1, com o número de árvores por clone e os valores de mínimo, médio e máximo para as variáveis idade, altura total (HT), diâmetro a 1,30 m do solo (DAP) e altura dominante (HD), totalizando 372 árvores.

De acordo com os dados da Tabela 1, os quatro clones utilizados neste estudo apresentam potencial produtivo (em termos volumétricos) relativamente semelhante, dado que não houve grande variação nos valores de altura, DAP e altura dominante entre eles. A maior variação foi observada entre o clone V3 e os demais, para os níveis máximos de cada característica medida. Esta variação é explicada pela menor idade máxima de amostragem deste material genético em relação aos outros 3 clones.

\section{Variações na densidade básica}

Os resultados de densidade básica obtidos nas análises dos discos retirados na altura do DAP das árvores e determinados pelo método da balança hidrostática apresentaram valor médio de $0,470 \mathrm{~g} / \mathrm{cm}^{3}$ para toda a população amostrada, com mínimo de $0,373 \mathrm{~g} / \mathrm{cm}^{3}$ e máximo de $0,561 \mathrm{~g} / \mathrm{cm}^{3}$, como pode ser observado na Figura 1.

Foi verificada tendência de aumento da densidade básica em função do aumento da idade das árvores, para todos os genótipos. Esta constatação está de acordo com vários autores que relataram relação positiva 
TABELA 1: Número de árvores amostradas por clone e os valores de mínimo, médio e máximo para 5 variáveis das populações clonais: idade, altura total (HT), diâmetro a 1,30 m do solo (DAP) e altura dominante (HD).

TABLE 1: Number of trees sampled per clone and minimum values, average and maximum for five variables of clonal populations: age, total height (HT), diameter at $1.30 \mathrm{~m}$ above the ground (DBH) and dominant height (HD).

\begin{tabular}{ccccccccccccccc}
\hline \multirow{2}{*}{ Clone } & \multirow{2}{*}{$\begin{array}{c}N^{o} \text { de } \\
\text { árvores }\end{array}$} & \multicolumn{3}{c}{ Idade $(\mathrm{anos})$} & \multicolumn{4}{c}{ HT $(\mathrm{m})$} & \multicolumn{4}{c}{ DAP $(\mathrm{cm})$} & \multicolumn{3}{c}{ HD $(\mathrm{m})$} \\
\cline { 2 - 14 } & Mín & Med & Máx & Mín & Med & Máx & Mín & Med & Máx & Mín & Med & Máx \\
\hline C2 & 80 & 1,9 & 4,3 & 7,6 & 13,4 & 21,8 & 32,9 & 8,3 & 13,8 & 21,6 & 14,8 & 23,7 & 31,7 \\
C4 & 120 & 2,1 & 4,4 & 7,7 & 9,7 & 22,2 & 35,5 & 8,0 & 13,9 & 23,2 & 13,5 & 24,3 & 32,4 \\
P95 & 80 & 2,0 & 4,4 & 7,7 & 11,1 & 22,5 & 32,2 & 7,6 & 13,5 & 21,8 & 17,0 & 24,6 & 32,2 \\
V3 & 92 & 2,0 & 4,2 & 5,5 & 12,8 & 22,4 & 29,2 & 6,7 & 13,0 & 20,5 & 16,7 & 24,8 & 30,3 \\
\hline
\end{tabular}

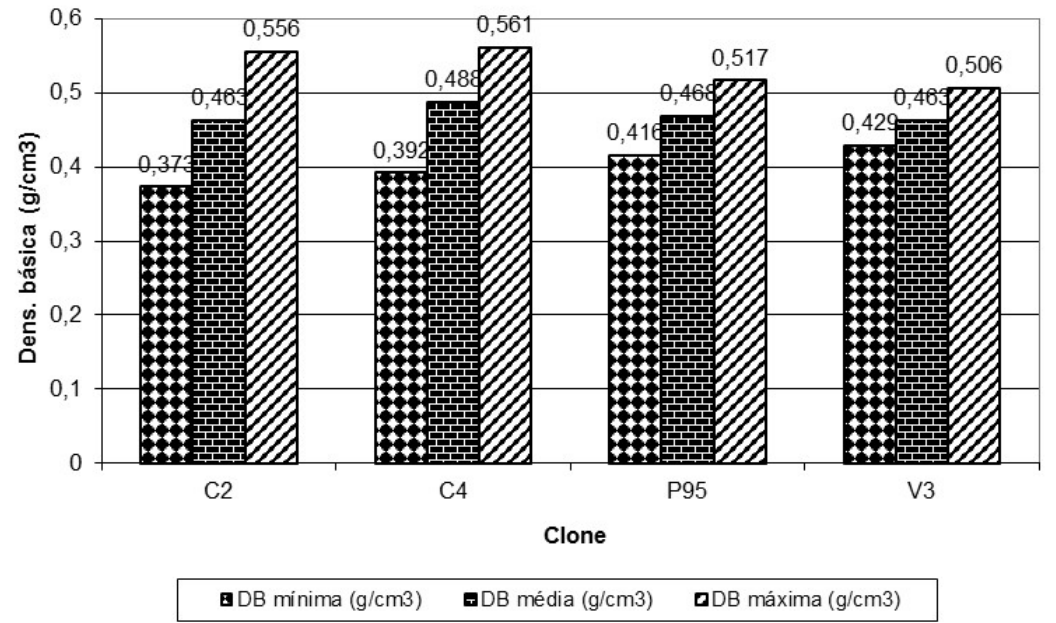

FIGURA 1: Valores mínimos, médios e máximos de densidade básica de discos na altura do DAP (diâmetro a 1,30 $\mathrm{m}$ do solo) para os 4 genótipos avaliados.

FIGURE 1: Minimum, average and maximum density of disks at the height of the DBH (at 1,30 $\mathrm{m}$ absore a roand) for the four genotypes.

da densidade básica da madeira de Eucalyptus com a idade, como, por exemplo, Vital (1987), Trugilho, Lima e Mendes (1996), Trugilho et al. (1997), Lopes e Garcia (2002), Thiersch (2002), Silva et al. (2004) e Eisfeld et al. (2009).

\section{Resultados de amplitude de resistência}

Os valores médios de amplitude de resistência dos 4 clones (AMP_100), registrados pelo Resistógrafo, variaram de 4,99 a 54,41\%, com média geral de 15,24\%. Lima et al. (2006), avaliando clones aos 7 anos de idade, obtiveram valores de amplitude entre 8,53 e 17,92\%. Em outro estudo, Lima et al. (2007) avaliaram 7 clones híbridos aos 16 anos de idade e encontraram valores de amplitude variando de 12,00 a 33,00\%. Andrade et al. (2008) avaliaram árvores aos 3 anos de idade e registraram amplitudes variando de 8,02 a $25,27 \%$.

A maior variação entre menor e maior valor de amplitude de resistência encontrada neste estudo em relação aos outros trabalhos citados, pode ser explicada pelo fato de que este contempla variações nas 
idades das árvores avaliadas, enquanto aqueles utilizaram uma idade fixa em seus estudos. Adicionalmente, a possível diferença entre os equipamentos utilizados e as regulagens destes, contribuem para a explicação desta variação.

\section{Equações selecionadas para estimativa da densidade básica}

Após o ajuste de vários modelos e testes de precisão dos mesmos, foi selecionado um conjunto de 20 equações lineares simples e múltiplas que apresentaram as melhores medidas de precisão na predição da densidade básica de cada genótipo e do conjunto dos 4 clones. Estas equações são apresentadas na Tabela 2.

As equações selecionadas apresentaram variações na precisão em função das varáveis explicativas utilizadas. Quando foi utilizada apenas a amplitude média de resistência à perfuração dada pelo Resistógrafo como variável independente para explicar a variação da densidade básica da população de estudo, a melhor forma de apresentação foi sempre a AMP_100. Este fato é um indicativo de que os tratamentos aplicados aos dados (eliminação de valores de amplitude nos percentis extremos ou eliminação pela técnica do "gráfico de caixa") não melhoraram o poder explicativo do Resistógrafo, e que estes na verdade fazem parte da variação natural da madeira.

A modelagem da densidade básica, utilizando apenas a amplitude média de resistência à perfuração dada pelo Resistógrafo, propiciou seleção de equações com coeficiente de determinação corrigido $\left(\mathrm{R}_{\mathrm{aj}}^{2}\right)$ de $19,47 \%$ para o clone V3, de 42,63\% para o clone P95, de 52,65\% para o clone C4 e de 55,76\% para o clone C2, sendo este coeficiente de $45,95 \%$ para a equação de predição para o conjunto dos 4 clones avaliados. Ao avaliar o poder preditivo da amplitude de resistência à perfuração com base no erro padrão residual das equações, verifica-se que este variou de $0,0299 \mathrm{~g} / \mathrm{cm}^{3}$ (ou $6,43 \%$ ) para o clone $\mathrm{C} 4$ a $0,0191 \mathrm{~g} / \mathrm{cm}^{3}$ (ou $4,05 \%$ ) para o clone P95, sendo $0,0262 \mathrm{~g} / \mathrm{cm}^{3}$ (ou 5,62\%) para os 4 clones.

Lima et al. (2007) ajustaram um modelo linear simples em função apenas da amplitude para 7 clones, em somente uma idade, e obtiveram um coeficiente de determinação igual a $64,00 \%$, com erro padrão da regressão igual a $0,036 \mathrm{~g} / \mathrm{cm}^{3}$. Em outro estudo, Lima et al. (2006) ajustaram modelos lineares simples com base no Resistógrafo para predição de densidade de 2 clones aos 7 anos de idade e obtiveram equações com $\mathrm{R}^{2}$ variando de $49,90 \%$ a $65,90 \%$ e erro padrão de $0,014 \mathrm{~g} / \mathrm{cm}^{3}$ a $0,012 \mathrm{~g} / \mathrm{cm}^{3}$. Essas variações foram obtidas com os mesmos clones, mas tomando medidas do Resistógrafo em direções diferentes na árvore (Norte-Sul e Sul-Norte), e também com a média de amplitude nas diferentes posições.

A inclusão de variáveis dendrométricas, associando-as aos dados do Resistógrafo (amplitude), melhorou a precisão das equações selecionadas para cada um dos 4 genótipos avaliados e também para o conjunto deles, propiciando aumento do coeficiente de determinação e redução do erro padrão de predição. Esses ganhos foram verificados com inclusão de variáveis de fácil obtenção, associadas ao indivíduo (DAP, idade e altura total) e aumentados ao acrescentar variáveis relacionadas à população (altura média dominante e diâmetro médio quadrático).

Ao trabalhar a modelagem da densidade básica eliminando os dados do Resistógrafo (amplitude média) e usando apenas variáveis dendrométricas, verificou-se comportamento diferente do grupo dos 4 clones em relação a cada um isoladamente. Para o conjunto dos clones (equação geral), o uso de somente dados dendrométricos permitiu o ajuste de uma equação que apresentou ganhos sobre aquela cuja única variável preditora foi a amplitude de resistência à perfuração dada pelo Resistógrafo. Já para cada clone em separado, as variáveis dendrométricas propiciaram melhor precisão não só em relação à amplitude média como única variável, mas também a esta acrescentada das variáveis do indivíduo.

Voltando à análise das equações selecionadas para a predição da densidade básica geral (os 4 clones ao mesmo tempo), tem-se que a que propiciou as melhores estimativas da densidade básica foi:

$$
D B=0,3990+0,00008129 \times\left(A M P \_100\right)^{2}-21,55 \times\left(\frac{1}{H D^{2}}\right)+0,2159 \times\left(\frac{I D}{H D}\right)+0,7264 \times\left(\frac{1}{D G}\right)+\varepsilon_{i}
$$

Em que DB é a densidade básica média da árvore $\left(\mathrm{g} / \mathrm{cm}^{3}\right)$, AMP_100 é a média de amplitude de resistência à perfuração coletada pelo Resistógrafo (\%), HD é a altura média das árvores dominantes (m) e ID é a idade da árvore (anos) e DG é o diâmetro médio quadrático da população onde a árvore avaliada se insere. 
TABELA 2: Equações selecionadas para predição da densidade básica dos clones C2, C4, P95, V3 e geral (todos os clones), com as respectivas estimativas dos parâmetros e medidas de precisão.

TABLE 2: Selected equations to predict the density of clones C2, C4, P95, V3, and general (all clones), with their parameter estimates and accuracy measures.

\begin{tabular}{|c|c|}
\hline Clone & Equações selecionadas \\
\hline \multirow{8}{*}{$\mathrm{C} 2$} & $\log (D B)=-0,45293-6,23721 \times\left(\frac{1}{A M P \_100}\right)+23,96093 \times\left(\frac{1}{A M P \_100^{2}}\right)+\varepsilon_{i}$ \\
\hline & Syx $\left(\mathrm{g} / \mathrm{cm}^{3}\right)=0,0296$ \\
\hline & $\log (D B)=-0,848531+0,089751 \times \log \left(A M P \_100\right)-0,040788 \times\left(\frac{D A P}{I D}\right)+\varepsilon_{i}$ \\
\hline & Syx $\left(\mathrm{g} / \mathrm{cm}^{3}\right)=0,0221$ \\
\hline & $\log (D B)=-0,313785+0,002776 \times\left(A M P_{-} 100\right)-7,116263 \times\left(\frac{1}{H D}\right)+1,074338 \times\left(\frac{I D}{H D}\right)-0,025168 \times(D G)+\varepsilon_{i}$ \\
\hline & Syx $\left(\mathrm{g} / \mathrm{cm}^{3}\right)=0,0179$ \\
\hline & $\log (D B)=-0,51664+25,39691 \times\left(\frac{1}{D G^{2}}\right)-8,12747 \times\left(\frac{1}{H D}\right)-0,02553 \times\left(\frac{H D}{I D}\right)+0,05905 \times \log (D A P)+\varepsilon_{i}$ \\
\hline & Syx $\left(\mathrm{g} / \mathrm{cm}^{3}\right)=0,0189$ \\
\hline \multirow{8}{*}{$\mathrm{C} 4$} & $\log (D B)=-1,25151+0,18498 \times \log \left(A M P \_100\right)+\varepsilon_{i}$ \\
\hline & Syx $\left(\mathrm{g} / \mathrm{cm}^{3}\right)=0,0299$ \\
\hline & $\log (D B)=-1,26465+0,09858 \times \log \left(A M P_{-} \_00\right)+0,13412 \times \log (D A P)-0,03205 \times\left(\frac{D A P}{I D}\right)+\varepsilon_{i}$ \\
\hline & Syx $\left(\mathrm{g} / \mathrm{cm}^{3}\right)=0,0245$ \\
\hline & $\log (D B)=-0,377443+0,005635 \times\left(A M P_{-} 100\right)+1,055221 \times\left(\frac{1}{I D}\right)-8,359632 \times\left(\frac{1}{H D}\right)-0,045061 \times\left(\frac{D G}{I D}\right)-0,038352 \times\left(\frac{H D}{I D}\right)+\varepsilon_{i}$ \\
\hline & Syx $\left(\mathrm{g} / \mathrm{cm}^{3}\right)=0,0217$ \\
\hline & $D B=0,356521+0,045001 \times(I D)-0,007555 \times(H D)-0,048039 \times\left(\frac{D G}{I D}\right)+0,044823 \times\left(\frac{H D}{I D}\right)+\varepsilon_{i}$ \\
\hline & Syx $\left(\mathrm{g} / \mathrm{cm}^{3}\right)=0,0234$ \\
\hline \multirow{9}{*}{ P95 } & $\log (D B)=-1,03690+0,10837 \times \log \left(A M P P_{-} 100\right)+\varepsilon_{i}$ \\
\hline & Syx $\left(\mathrm{g} / \mathrm{cm}^{3}\right)=0,0191$ \\
\hline & $\log (D B)=-1,05005+0,07605 \times \log \left(A M P \_100\right)+0,06851 \times \log (I D)+\varepsilon_{i}$ \\
\hline & Syx $\left(\mathrm{g} / \mathrm{cm}^{3}\right)=0,0161$ \\
\hline & $\log (D B)=-0,51398-0,81933 \times\left(\frac{1}{A M P \_100}\right)+1,03235 \times\left(\frac{1}{I D}\right)-127,49513 \times\left(\frac{1}{H D^{2}}\right)-0,05317 \times\left(\frac{H D}{I D}\right)+1,54040 \times\left(\frac{1}{D G}\right)+\varepsilon_{i}$ \\
\hline & Syx $\left(\mathrm{g} / \mathrm{cm}^{3}\right)=0,0140$ \\
\hline & $D B=0,050871-0,016848 \times(H D)+0,043759 \times(D G)+15,756850 \times\left(\frac{1}{D G}\right)-21,719118 \times\left(\frac{1}{H D}\right)+0,103751 \times\left(\frac{D G}{I D}\right)-0,069141 \times$ \\
\hline & $\left(\frac{H D}{I D}\right)+0,019075 \times \log (D A P)+\varepsilon_{i}$ \\
\hline & Syx $\left(\mathrm{g} / \mathrm{cm}^{3}\right)=0,0152$ \\
\hline
\end{tabular}

Continua... 
TABELA 2: Continuação...

TABLE 2: Continued...

Clone Equações selecionadas

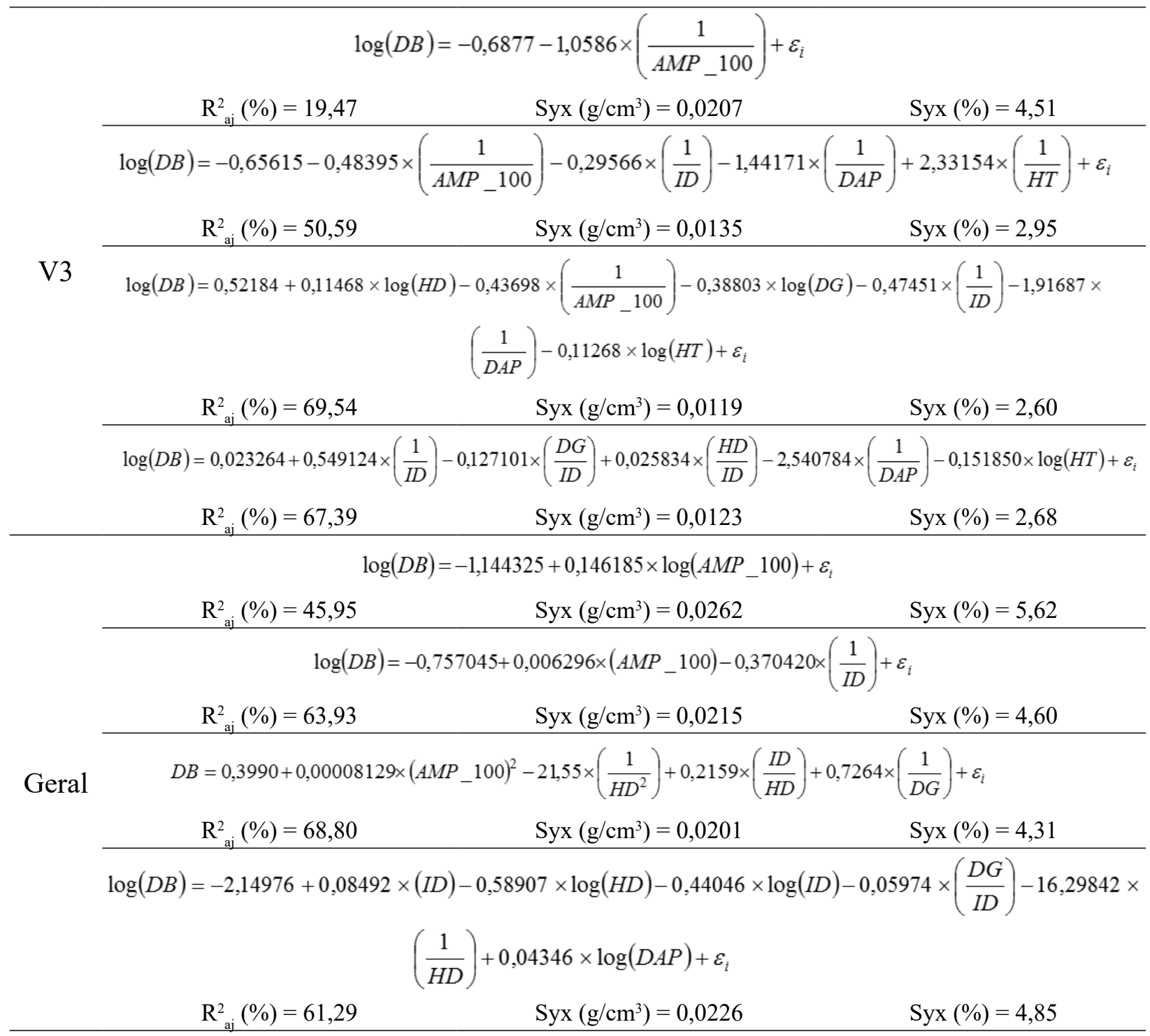

Em que: DB - densidade básica $\left(\mathrm{g} / \mathrm{cm}^{3}\right)$; AMP_100 - amplitude média dada pelo Resistógrafo na árvore (\%); ID - idade em anos; DAP - diâmetro a 1,30 m do solo (cm); HT - altura total das árvores (m); HD - altura média das árvores dominantes $(\mathrm{m})$; DG - diâmetro médio quadrático $\left(\mathrm{cm}^{2}\right) ; \mathrm{R}_{\text {aj }}^{2}-$ coeficiente de determinação ajustado e Syx erro padrão residual.

As medidas de precisão da equação acima revelam um erro padrão residual de $0,0201 \mathrm{~g} / \mathrm{cm}^{3}(4,31 \%)$ na predição da densidade média das árvores, com um coeficiente de determinação ajustado $\left(\mathrm{R}_{\text {aj }}^{2}\right)$ de $68,80 \%$, indicando ser este o poder explicativo dos dados do Resistógrafo associados às variáveis citadas sobre a variação da densidade básica nas populações dos 4 genótipos avaliados.

Na Tabela 3 são apresentadas as estimativas dos parâmetros da equação, com a estatística " $t$ " de Student para cada um deles.

A estatística " $t$ ", apresentada na Tabela 3, mostra que as variáveis que compõem a equação são altamente significativas, indicando a importância destas na explicação das variações observadas na variável resposta.

A Figura 2 mostra a análise gráfica da melhor equação selecionada para predição da densidade básica 
dos 4 genótipos avaliados (equação geral), em que "dbm" é a densidade básica média observada e "dbm est" é a densidade básica média estimada, ambas em $\mathrm{g} / \mathrm{cm}^{3}$.

TABELA 3: Estimativas da significância dos parâmetros da melhor equação linear múltipla selecionada para os 4 clones.

TABLE 3: Estimates of the parameter significances of the best multiple linear equation selected for the four clones.

\begin{tabular}{lcccc}
\hline & Estimativa & Erro padrão & Valor t & $\operatorname{Pr}(>|\mathrm{t}|)$ \\
\hline$\beta 0$ & 0,3990 & 0,01818 & 21,944 & $<2 \mathrm{e}-16^{* * *}$ \\
$\beta 1$ & 0,00008129 & 0,000008111 & 10,022 & $<2 \mathrm{e}-16^{* * *}$ \\
$\beta 2$ & $-21,55$ & 2,221 & $-9,704$ & $<2 \mathrm{e}-16^{* * *}$ \\
$\beta 3$ & 0,2159 & 0,03717 & 5,808 & $1,89 \mathrm{e}-08^{* * *}$ \\
$\beta 4$ & 0,7264 & 0,2395 & 3,033 & $0,00267 * *$
\end{tabular}

Em que: *** e ** $=$ significância a 0,001 e 0,01 , respectivamente.

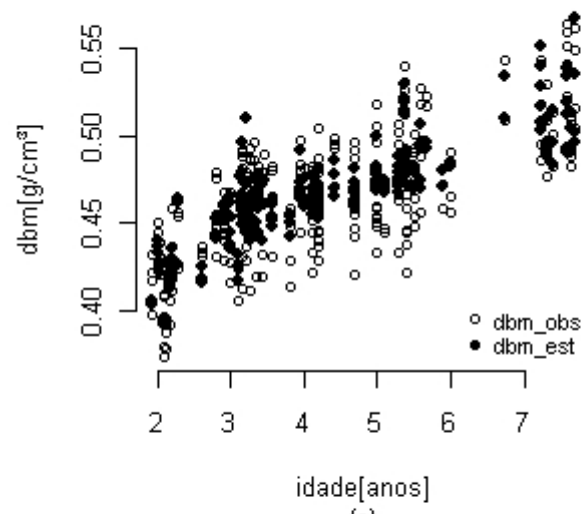

(a)

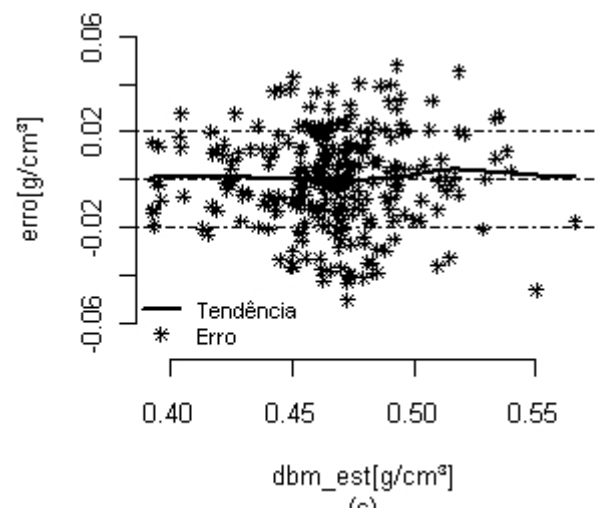

(c)

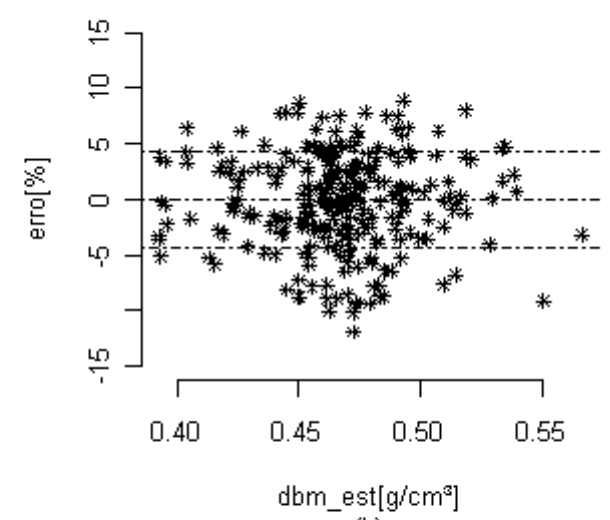

(b)

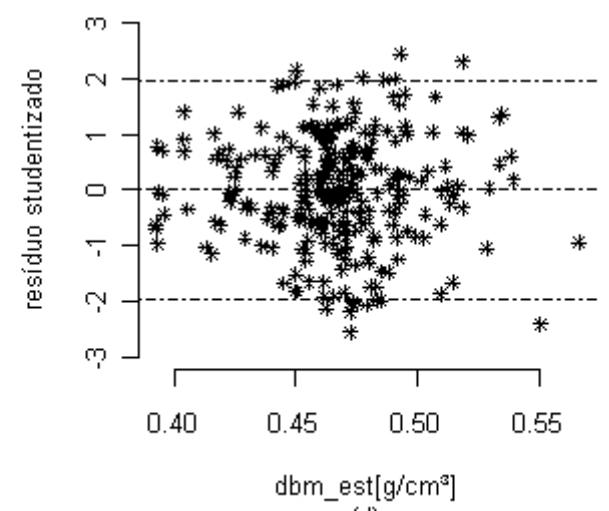

(d)

FIGURA 2: Análise gráfica da melhor equação selecionada para o conjunto dos 4 clones: (a) densidade estimada versus observada, (b) dispersão dos resíduos em percentagem, (c) dispersão dos resíduos em $\mathrm{g} / \mathrm{cm}^{3}$ e (d) dispersão dos resíduos "studentizados".

FIGURE 2: Graphic analysis of the best fit equation selected for all four clones: (a) observed versus estimated density, (b) dispersion of the residuals as a percentage, (c) dispersal of residuals in $\mathrm{g} / \mathrm{cm} 3$ and (d) dispersion of residuals "studentized". 
Houve boa sobreposição dos valores de densidade básica estimada em relação à densidade básica observada (a), e dispersão de resíduos relativamente homogênea em torno da média zero (b e c), não apresentando tendências importantes de erro de estimativa dentro da faixa de densidade básica identificada na população de estudo. Em (d) é apresentada a dispersão dos resíduos "studentizados", bem distribuída em torno da média zero, sem tendências evidentes, e com poucos dados fora da faixa de $95 \%$ de probabilidade pela estatística " $t$ " de Student.

\section{CONCLUSÕES}

A densidade básica média da população de estudo, com base na análise de um disco por árvore na altura do DAP, apresentou tendência de aumento em função do aumento da idade das árvores.

Os valores médios de amplitude de resistência à perfuração dos 4 clones (AMP_100), registrados pelo Resistógrafo, variaram de 4,99 a 54,41\%, com média geral de $15,24 \%$.

A equação selecionada para predição da densidade básica do conjunto dos 4 clones, utilizando apenas a amplitude média de resistência à perfuração dada pelo Resistógrafo foi:

$$
\log (D B)=-1,144325+0,146185 \times \log \left(A M P_{-} 100\right)+\varepsilon_{i}
$$

Com $\mathrm{R}_{\text {aj }}^{2}$ de $45,95 \%$ e erro padrão residual igual a $\pm 5,62 \%$.

Equações ajustadas utilizando apenas dados dendrométricos relacionados ao indivíduo e à população, em detrimento do uso do Resistógrafo (amplitude média de resistência à perfuração), apresentaram melhor precisão na estimativa de densidade básica tanto para o conjunto dos 4 clones quanto para cada clone isoladamente. Assim, a equação ajustada apenas a partir de dados dendrométricos para o conjunto dos 4 clones foi:

$$
\log (D B)=-2,14976+0,08492 \times(I D)-0,58907 \times \log (H D)-0,44046 \times \log (I D)-0,05974 \times\left(\frac{D G}{I D}\right)-16,29842 \times\left(\frac{1}{H D}\right)+0,04346 \times \log (D A P)+\varepsilon_{i}
$$

Com $\mathrm{R}_{\text {aj }}^{2}$ de $61,29 \%$ e erro padrão residual igual a $\pm 4,85 \%$.

Entretanto, quando foram utilizados dados do Resistógrafo (amplitude média de resistência à perfuração) associados aos dados dendrométricos, foi possível ajuste de equações mais precisas para a estimativa da densidade básica dos clones, tanto isoladamente quanto em conjunto, indicando que o Resistógrafo tem potencial para uso como ferramenta na predição de densidade básica de clones de Eucalyptus.

Por fim, a melhor equação selecionada para predição da densidade básica para o conjunto dos 4 clones utilizados neste estudo foi obtida associando variáveis dendrométricas do nível indivíduo e do nível população, associadas aos dados de amplitude média de resistência à perfuração coletados pelo Resistógrafo:

$$
D B=0,3990+0,00008129 \times\left(A M P_{-} 100\right)^{2}-21,55 \times\left(\frac{1}{H D^{2}}\right)+0,2159 \times\left(\frac{I D}{H D}\right)+0,7264 \times\left(\frac{1}{D G}\right)+\varepsilon_{i}
$$

Com $\mathrm{R}_{\text {aj }}$ de $68,80 \%$ e com erro padrão residual igual a $\pm 4,31 \%$.

\section{REFERÊNCIAS}

ALZATE, S. B. A.; TOMAZELLO FILHO, M.; PIEDADE, S. M. F. Variação longitudinal da densidade básica da madeira de clones de Eucalyptus grandis Hill ex Maiden, E. saligna Sm e E. grandis x E. urophylla. Scientia Forestalis. Piracicaba, n. 68, p. 87-95, 2005.

ANDRADE, C. R. et al. Relação entre a densidade básica da madeira de clones de Eucalyptus e os ensaios não-destrutivos. In: ENCONTRO BRASILEIRO EM MADEIRAS E ESTRUTURAS DE MADEIRA. 11., 2008, Londrina. Anais... Londrina, 2008. 11 p.

EISFELD, R. et al. Modelagem da massa específica básica dos cavacos a partir de variáveis dendrométricas e da massa específica do disco. Floresta, Curitiba, v. 39, n. 4, p. 877-886, 2009.

FOELKEL, C. E.; MORA, E.; MENOCHELLI, S. Densidade básica: sua verdadeira utilidade como 
índice de qualidade da madeira de eucalipto para produção de celulose. In: CONGRESSO FLORESTAL BRASILEIRO, 6., Campos do Jordão. Anais... 1990, p. 719-728.

GOMES, A. F. Avaliação das características da madeira e da polpa de Eucalyptus mediante a aplicação de métodos não destrutivos na árvore viva. 2007. 124 f. Dissertação (Mestrado) - Universidade Federal de Lavras, Lavras, 2007.

HANSEN, C. P. Application of the pilodyn in Forest tree improvement. Denmark: Danida Forest seed Centre, 2000. (Series of Technical Notes, 55).

LIMA, J. T. et al. Adequação do Resistograph para a estimativa da densidade básica da madeira de Eucalyptus. In: ENCONTRO BRASILEIRO EM MADEIRAS E EM ESTRUTURAS DE MADEIRA EBRAMEM, 10., 2006, São Padro. Anais... São Pedro, 2006. 16 p.

LIMA, J. T. et al. Uso do Resistógrafo ${ }^{\circledR}$ para estimar a densidade básica e a resistência à perfuração da madeira de Eucalyptus, Scientia Forestalis, Piracicaba, n. 75, p. 85-93, 2007.

LOPES, G. A.; GARCIA, J. N. Densidade básica e umidade natural da madeira de Eucalyptus saligna Smith, de Itatinga, associadas aos padrões de casca apresentados pela população, Scientia Forestalis, Piracicaba, n. 62, p. 13-23, 2002.

LOPEZ, J.; GOMIDE, J. L.; PHILLIPS, R. Impacto da qualidade da madeira de eucalipto no desempenho financeiro de um modelo de fábrica brasileira de celulose. O Papel, São Paulo, v. 70, n. 7, p. 53-71, 2009.

QUEIROZ, S. C. S. et al. Influência da densidade básica da madeira na qualidade da polpa kraft de clones híbridos de Eucalyptus grandis W. Hill ex Maiden X Eucalyptus urophylla S. T. Blake, Revista Árvore, Viçosa, MG, v. 28, n. 6, p. 901-909, 2004.

RINN, F.; SCHWEINGRUBER, F. H.; SCHAR, E. Resistograph and X-ray density charts of wood comparative evaluation of drill resistance profiles and X-ray density charts of different wood species, Holzforschung, Berlin, v. 50, n. 4, p. 303-311, 1996.

SANTOS, C. R. Qualidade da madeira e sua influência nas características de papéis de imprimir \& escrever e tissue. 2002. 45 f. Monografia (Graduação) - Universidade Federal de Viçosa, Viçosa, MG, 2002.

SILVA, J. C. et al. Influência da idade e da posição radial na massa específica da madeira de Eucalyptus grandis Hill ex Maiden. Revista Floresta, Curitiba, v. 34, n. 1, p. 13-22, 2004.

THIERSCH. C. R. Modelagem da densidade básica, do volume e do peso seco para plantios de clones de Eucalyptus sp. 2002. 197 p. Dissertação (Mestrado em Engenharia Florestal) - Universidade Federal de Lavras, Lavras, 2002.

THIERSCH. C. R. et al. O uso de modelos matemáticos na estimativa da densidade básica da madeira em plantios de clones de Eucalyptus sp. Cerne, Lavras, v. 12, n. 3, p. 264-278, 2006.

TRUGILHO, P. F.; LIMA, J. T.; MENDES, L. M. Influência da idade nas características físico-químicas e anatômicas da madeira de Eucalyptus saligna, Cerne, Lavras, v. 2, n. 1, p. 94-111, 1996.

TRUGILHO, P. F. et al. Influência da idade nas características físicas, químicas e anatômicas da madeira de Eucalyptus grandis. In: IUFRO CONFERENCE ON SILVICULTURE AND IMPROVEMENT OF EUCALYPTS, 1997, Salvador. Anais... Colombo: EMBRAPA/CPNF, 1997. v. 3, p. 269-275.

TRUGILHO, P. F. et al. Utilização de métodos não destrutivos na avaliação de clones de Eucalyptus spp. In: ENCONTRO BRASILEIRO EM MADEIRAS E EM ESTRUTURAS DE MADEIRA, 10., 2006, São Pedro. Anais... São Pedro, 2006.

VITAL, B. R. Efeito da idade da árvore na densidade da madeira de Eucalyptus grandis cultivado na região do cerrado de Minas Gerais. Brasília: IBDF, 1987. (Boletim técnico, 8). 